\title{
Comparison of continuous subcutaneous insulin infusion and insulin glargine-based multiple daily insulin aspart injections with preferential adjustment of basal insulin in patients with type 2 diabetes
}

\author{
GUAN-QI GAO ${ }^{1}$, XUE-YUAN HENG ${ }^{2}$, YUE-LI WANG ${ }^{1}$, WEN-XIA LI ${ }^{1}$, \\ QING-YU DONG ${ }^{1}$, CUI-GE LIANG ${ }^{1}$, WEN-HUA DU ${ }^{1}$ and XIAO-MENG LIU ${ }^{1}$ \\ Departments of ${ }^{1}$ Endocrinology and ${ }^{2}$ Clinical Medicine, Linyi People's Hospital, Linyi, Shandong 276003, P.R. China
}

Received November 21, 2013; Accepted May 2, 2014

DOI: $10.3892 / \mathrm{etm} .2014 .1866$

\begin{abstract}
The purpose of this study was to evaluate and compare multiple daily injection (MDI) therapy of bolus insulin aspart and basal insulin glargine with continuous subcutaneous insulin infusion (CSII) with aspart in patients with type 2 diabetes mellitus (T2DM). It was assessed whether MDI was capable of controlling glycemic index with a higher efficacy than CSII by preferential adjustment of basal insulin with a lower total daily insulin dosage in T2DM. Two hundred patients with T2DM were enrolled in the study and randomly assigned to CSII $(n=100)$ and MDI $(n=100$; aspart immediately prior to each meal and glargine at bedtime) groups for 12 weeks of therapy. During the last week of each treatment period, the subjects wore a continuous glucose monitoring system for 2-3 days. The dosage of basal insulin was preferentially adjusted to control prior-meal blood glucose levels, and the characteristics of insulin dosage were analyzed. No statistically significant differences were observed between the two groups in hemoglobin Alc (HbAlc), which dropped from $10-11 \%$ prior to therapy to $7-7.5 \%$ after 12 weeks. After 12 weeks, good glycemic level control was achieved in all patients in the MDI and CSII groups. A statistically significant difference in the dose of insulin between the CSII and MDI groups was observed $(\mathrm{P}<0.001)$. In conclusion, no significant differences were found between the two therapies in the incidence of hypoglycemia and $\mathrm{HbAlc}$ for the 12 weeks. The basal insulin dosage was significantly decreased in the MDI group compared with that in the CSII group, but the CSII group was superior to MDI group in decreasing fasting blood glucose
\end{abstract}

Correspondence to: Dr Guan-Qi Gao, Department of Endocrinology, Linyi People's Hospital, 27 Jiefang Road, Linyi, Shandong 276003, P.R. China

E-mail: gaoguanqi2013@126.com

Key words: type 2 diabetes, insulin aspart, glargine insulin, hypoglycemia and shortening the time required for hypoglycemia to meet the targeted level.

\section{Introduction}

Continuous subcutaneous insulin infusion (CSII) therapy with an external pump and multiple daily injection (MDI) therapy are two of the currently selected methods of insulin treatment for diabetes. MDI therapy for diabetes requires bolus injections of rapid or short-acting insulin prior to each meal and a long-acting insulin injection once or twice per day for basal insulin coverage. Control of postprandial glycemia with rapid-acting insulin has been shown to be more effective than that with normal human insulin $(1,2)$. Long-acting insulin, such as insulin glargine, is suitable as a basal insulin therapy in diabetes $(3,4)$. CSII therapy produces a higher efficacy than MDI and improvements in insulin pump technology have resulted in an increase in patient preference (5-10). However, occasionally patients may have to temporarily discontinue CSII therapy due to skin problems, pump malfunction or physical activity. It has been shown that MDI therapy is at least equivalent in its action to CSII (10).

Type 2 diabetes mellitus (T2DM) is characterized by a progressive reduction in $\beta$-cell secretion of insulin and mass together with insulin resistance (11). The incidence rate of T2DM has been reported to be $9.7 \%$ in China (12) and Chinese patients with T2DM have shown more significant $\beta$-cell deterioration than patients of other genealogies (13). Insulin preparation and medication have undergone rapid progress; however, the amount of insulin injected falls short of what is physiologically secreted in individuals without T2DM. Furthermore, insulin therapy may cause weight increases, hypoglycemia and iatrogenic hyperinsulinemia, which can increase insulin resistance and the potential risks of vascular disease (14). High doses of insulin can induce insulin resistance in diabetic rats, whilst intermediate doses can maximally improve insulin sensitivity (10); therefore, studies investigating the optimal insulin dosage are required. Numerous investigations in patients with type 1 diabetes mellitus (T1DM) have shown that CSII therapy was more efficacious than MDI therapy $(15,16)$, since CSII decreased the dose of insulin required to a greater 
degree (17-19). Phillip et al (20) demonstrated that the higher dose of insulin required with MDI in patients with T1DM, the more marked the insulin decrease subsequent to switching to CSII. Conversely, Monami et al (21) showed by meta-analysis of insulin replacement in patients with T2DM that the daily dose of insulin with CSII was not significantly different from that with MDI therapy. Similarly, a study of Indian patients with T2DM showed no significant differences in the total daily dose of insulin when therapy was transitioned from MDI to CSII for six months (22).

The dysfunction of $\beta$ cells has been found to be a major contributing factor in Chinese patients with T2DM (13); therefore, insulin remains the first choice of therapy. However, as previously discussed, this treatment may result in weight increase, hypoglycemia and iatrogenic hyperinsulinemia, thus increasing the potential risks of insulin resistance, vascular disease and sleep apnea syndrome (14,23-26). Bruttomesso et al $(15,17)$ and Hoogma et al $(27)$ found that patients with T1DM using CSII required a lower insulin dosage, as compared with those using MDIs. In the present study, it was hypothesized that insulin dosage adjustment may also improve therapy for patients with T2DM. In China, MDI therapy of bolus insulin aspart and basal insulin glargine and CSII are mainly used for glycemic control and treatment of T2DM in hospitalized patients with T2DM. There currently have not been enough relevant investigations demonstrating the optimal insulin dosage in Chinese patients with T2DM. In this study, the insulin dosage characteristics of 200 hospitalized patients with T2DM who were treated with CSII and MDI therapy were investigated.

\section{Patients and methods}

Subjects. Two hundred patients with T2DM (80 females and 120 males) were recruited for this study. All subjects were hospitalized between January 2011 and July 2013 in the Department of Endocrinology at Linyi People's Hospital (Linyi, China). The diagnosis of T2DM was established on the basis of the 1999 Diabetes Diagnostic Criteria of the World Health Organization. Subjects hospitalized for insulin treatment due to poor glycemic control and newly diagnosed patients with high blood glucose were enrolled. Subjects prescribed other oral hypoglycemia agents, with the exception of metformin, were excluded and none of the subjects received basal insulin regimen intensification prior the antidiabetic treatment.

Subjects with impaired renal or hepatic function and those who were pregnant or breast-feeding, had malignancies, impaired cardiac function, hypoglycemia unawareness, acute infection or acute complications (such as diabetic nonketotic hyperosmolar coma, diabetic ketoacidosis and diabetic lactic acidosis) were excluded from the study. Investigators and patients were blinded to the therapy sequence up to the point of subject randomization. Subjects were randomly divided into a CSII or MDI group, according to their hospitalization number. One hundred subjects (60 males and 40 females) were randomly assigned to continued therapy with CSII and 100 subjects (60 males and 40 females) to therapy with MDI for 12 weeks. Subjects in the MDI group were administered insulin aspart immediately prior to each meal and then insulin glargine before bed. In the CSII group, all patients received insulin; 32 had peripheral neuropathy, 18 had retinopathy and six had large vessel diseases. In the MDI group, all patients received insulin; 31 subjects had peripheral neuropathy, 18 had retinopathy and seven had large vessel diseases.

Ethics statement. The investigation was approved by the Medical Ethics Committee of Linyi People's Hospital. All subjects were patients admitted to the Department of Endocrinology and all patients signed a consent form allowing their information to be stored in the hospital database and used for this study. The consent form was approved by the Medical Ethics Committee of Linyi People's Hospital.

Investigation design and method. No oral hypoglycemic drugs, with the exception of metformin, were used during the insulin treatment in the present study. The initial dosage for all subjects was $0.3-0.4 \mathrm{IU} / \mathrm{kg} / \mathrm{day}$. The MDI group was treated with insulin aspart (Novo Nordisk, Bagsværd, Denmark) injected subcutaneously prior to each of the three meals in the day, as well as a single basal bedtime injection of insulin glargine (Lantus ${ }^{\circledR}$, Sanofi-Aventis Pharmaceuticals, Mumbai, India) daily, for 12 weeks. The initial glargine-based insulin injections accounted for $60 \%$ of the total daily dose and the insulin aspart accounted for $40 \%$. The CSII group was treated with insulin aspart using insulin pumps (Medtronic, Northridge, CA, USA). In the CSII group, the initial basal dose, which accounted for $60 \%$ of the total daily amount, was divided into the following four periods of the day: midnight-4:00 a.m., 4:00-9:00 a.m., 9:00 a.m.-9:00 p.m. and 9:00 p.m.-midnight. The three pre-meal doses together accounted for $40 \%$ of the total daily dose and were administered for 12 weeks. Blood glucose levels were monitored using a stable blood glucose-monitoring device from LifeScan (Johnson \& Johnson Company, Milpitas, CA, USA). Blood glucose levels were measured from finger-stick blood samples eight times per day (before each of the three meals, $2 \mathrm{~h}$ later and at 10:00 pm and 3:00 am). The diet of all subjects was regulated according to the China Guideline for T2DM. The basal insulin dose was preferentially adjusted to control the blood glucose when the pre-meal blood glucose level was $>9 \mathrm{mmol} / \mathrm{l}$ or the postprandial glucose $(\mathrm{PBG})$ was $<6 \mathrm{mmol} / \mathrm{l}$ according to the features of the PBG state in patients with T2DM $(15,26)$. Pre-meal doses were distributed evenly among the three meals. Basal insulin doses were titrated to target fasting glucose between 4.0 and $7.0 \mathrm{mmol} / \mathrm{l}$. Pre-meal insulin doses were adjusted according to 2 -h PBG levels to achieve the target of $\leq 11.0 \mathrm{mmol} / \mathrm{l}$. If the subject achieved two consecutive days at this level, the length of time required to achieve the target, total daily insulin doses, daily basal insulin doses, blood glucose fluctuations and hypoglycemic episodes were calculated. Hypoglycemic episodes were classified as 'severe hypoglycemia' when patients were not able to treat the episode themselves and blood glucose was $\leq 3.9 \mathrm{mmol} / \mathrm{l}$, 'symptomatic hypoglycemia' when patients were able to treat the episode and blood glucose was $\leq 3.9 \mathrm{mmol} / \mathrm{l}$, and 'relative hypoglycemia' when patients exhibited symptoms of hypoglycemia but blood glucose was either $>3.9 \mathrm{mmol} / \mathrm{l}$ or not measured (28). Hypoglycemic episodes were evaluated as all events (all episodes occurring over a $24-\mathrm{h}$ period) and nocturnal events (episodes occurring between 11:00 p.m. and 6:00 a.m.). Once glycemic control was stabilized for 3 days, the daily insulin dose was calculated when the pre-meal glucose and 
PBG were $<7.01$ and $11.09 \mathrm{mmol} / \mathrm{l}$, respectively. The state of hypoglycemia was defined as blood glucose levels $\leq 3.9 \mathrm{mmol} / \mathrm{l}$ or where symptoms of hypoglycemia resolved with administration of oral carbohydrates, and a decrease in basal insulin or regular insulin dose according to pre-meal glucose and PBG.

Statistical analysis. All statistical data analyses were performed using SPSS version 13. (SPSS, Inc., Chicago, IL, USA). Descriptive data analyses of the qualitative variables were performed with proportions and percentages. Quantitative variables are presented as the mean \pm standard deviation. The variables were analyzed with the independent samples t-test between the CSII and MDI groups. A value of $\mathrm{P}<0.05$ was considered to be statistically significant.

\section{Results}

Clinical data. No statistically significant differences were observed between the CSII and MDI groups in gender, age, hemoglobin A1c (HbA1c), fasting serum C-peptide, body mass index, fasting blood glucose (FBG) or other clinical data $(\mathrm{P}>0.05)$ (Table I).

Insulin doses and incidence of hypoglycemia. Neither the MDI nor the CSII groups had severe hypoglycemic episodes during the treatment duration, and no statistically significant differences were observed in nocturnal hypoglycemic episodes. Good glycemic level control was achieved in the 100 subjects in the MDI group after $6.88 \pm 2.31$ days. The mean total daily dosage of insulin was $37.12 \pm 10.18 \mathrm{IU}(0.58 \pm 0.17 \mathrm{IU} / \mathrm{kg} /$ day $)$, and the total daily basal and bolus doses were $19.35 \pm 7.84$ and $17.55 \pm 3.52 \mathrm{IU}(50.80 \pm 8.32$ and $49.11 \pm 8.32 \%$ of the total daily dose), respectively. Good glycemic level control was achieved in the 100 subjects in the CSII group after $5.43 \pm 2.30$ days. The decrease in HbA1c in the two groups was reached earlier in patients in the CSII group compared with those in the MDI group. The mean total daily insulin dose in the CSII group was $31.68 \pm 8.88 \mathrm{IU}(0.48 \pm 0.16 \mathrm{IU} / \mathrm{kg} /$ day $)$, and the total daily basal and bolus doses were $22.77 \pm 7.65$ and $9.78 \pm 2.74$ IU $(69.13 \pm 6.99$ and $30.87 \pm 6.99 \%$ of the total daily dose), respectively (Table II). A significant difference in the dose of insulin was observed between the CSII and MDI groups $(\mathrm{P}<0.001)$. Insulin requirements decreased $19.12 \pm 2.3 \%$ after 12 weeks for good glycemic level control in the two groups. Significant differences were found between the two groups in the total dose of insulin and the basal and bolus doses of insulin per day $(\mathrm{P}<0.001)$ (Fig. 1 and Table II). The incidence of hypoglycemia was $5.93 \%$ in the MDI group and $1.62 \%$ in the CSII group $(\mathrm{P}<0.01)$ (Table II).

Treatment efficacy. The mean HbA1c showed a statistically significant decrease during the course of the experiment for the MDI and CSII groups (Table III). In the MDI group, mean HbAlc decreased from $10.79 \pm 1.42$ to $7.51 \pm 1.28 \%$. In the CSII group, mean HbA1c decreased from $10.86 \pm 1.36$ to $7.11 \pm 1.32 \%$. No significant differences were identified between the groups.

The mean FBG levels in the two groups showed statistically significant baseline to end-point decreases, from $8.61 \pm 3.12$ to $6.76 \pm 1.13 \mathrm{mmol} / 1$ in the CSII group and from $8.68 \pm 3.32$ to $6.85 \pm 1.26 \mathrm{mmol} / \mathrm{l}$ in the MDI group ( $\mathrm{P}<0.005$ for both). No
Table I. Demographic characteristics of the subjects.

\begin{tabular}{lcc}
\hline & \multicolumn{2}{c}{ Group } \\
\cline { 2 - 3 } Parameter & MDI & CSII \\
\hline Male/female (n/n) & $60 / 40$ & $60 / 40^{\mathrm{a}}$ \\
Newly diagnosed patients (n) & 36 & $36^{\mathrm{a}}$ \\
Duration of diabetes (years) & $6.78 \pm 5.71$ & $6.89 \pm 5.79^{\mathrm{a}}$ \\
Age (years) & $51.38 \pm 11.73$ & $50.58 \pm 12.67^{\mathrm{a}}$ \\
BMI $\left(\mathrm{kg} / \mathrm{m}^{2}\right)$ & $24.41 \pm 3.62$ & $24.89 \pm 3.47^{\mathrm{a}}$ \\
HbAlc $(\%)$ & $10.86 \pm 1.36$ & $10.79 \pm 1.42^{\mathrm{a}}$ \\
FBG $(\mathrm{mmol} / \mathrm{l})$ & $7.61 \pm 3.12$ & $8.68 \pm 3.32^{\mathrm{a}}$ \\
2-h PBG (mmol/l) & $15.42 \pm 4.78$ & $15.60 \pm 5.71^{\mathrm{a}}$ \\
FCP (ng/ml) & $0.71 \pm 0.44$ & $0.72 \pm 0.45^{\mathrm{a}}$ \\
\hline
\end{tabular}

Descriptive data are expressed as the mean \pm standard deviation ${ }^{\mathrm{a}} \mathrm{P}>0.05$ versus MDI group. HbA1c, hemoglobin A1c; BMI, body mass index; FBG, fasting blood glucose; PBG, postprandial blood glucose; FCP, fasting C-peptide; MDI, multiple daily injection; CSII, continuous subcutaneous insulin infusion.

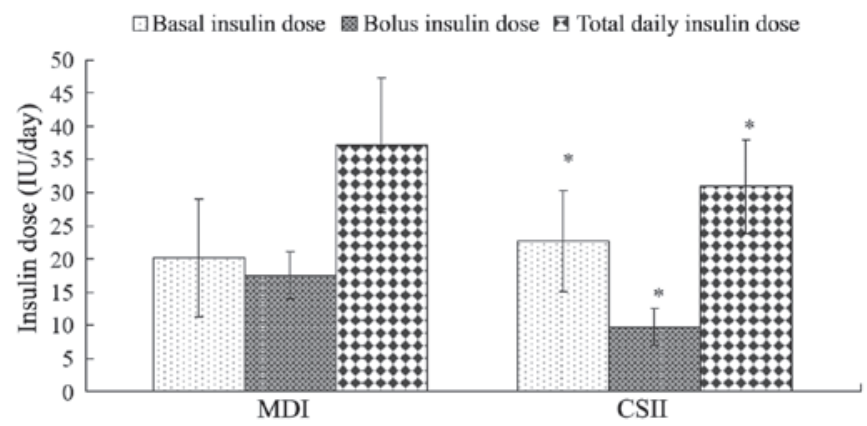

Figure 1. Insulin dose distribution of the MDI and CSII groups. "P $<0.05$ versus MDI group. MDI, multiple daily injection; CSII, continuous subcutaneous insulin infusion.

statistically significant differences in baseline to end-point FBG level decreases were observed between the two groups (P>0.05) (Table III).

The mean 2-h PBG level decreased significantly from $15.42 \pm 4.78 \mathrm{mmol} / \mathrm{l}$ at baseline to $9.87 \pm 2.63 \mathrm{mmol} / \mathrm{l}$ at the end-point in the CSII group and from $15.60 \pm 5.71$ to $9.95 \pm 2.16 \mathrm{mmol} / 1$ in the MDI group ( $\mathrm{P}<0.005$ for both). No statistically significant differences in baseline to end-point 2-h PBG level decreases were observed between the two groups (P>0.05) (Table III).

Changes in insulin doses. The total daily insulin dose in the CSII group decreased from $0.48 \pm 0.16$ to $0.39 \pm 0.23 \mathrm{IU} / \mathrm{kg} /$ day after 12 weeks $(-0.09 \pm 0.22 \mathrm{IU} / \mathrm{kg} /$ day $)$, and from $0.58 \pm 0.17$ to $0.47 \pm 0.19 \mathrm{IU} / \mathrm{kg} /$ day after 12 weeks in the MDI group $(-0.11 \pm 0.21 \mathrm{IU} / \mathrm{kg} /$ day $)$; however, the basal and bolus insulin doses as percentages of the total daily dose remained unchanged at the end of the 12-week period (Table IV).

Blood glucose levels. Blood glucose levels in the CSII group were lower than those in the MDI group $(\mathrm{P}>0.05)$ at each time-point; 
Table II. Comparison of insulin doses upon achievement of good blood glucose control at the start of the 12 weeks of therapy.

\begin{tabular}{lccccc}
\hline Group & $\mathrm{n}$ & Rate (IU/kg/day) & Basal insulin dose (\%) & Bolus insulin dose (\%) & Incidence of hypoglycemia (\%) \\
\hline CSII & 100 & $0.48 \pm 0.16^{\mathrm{a}}$ & $69.13 \pm 6.99^{\mathrm{a}}$ & $30.87 \pm 6.99^{\mathrm{a}}$ & $1.62^{\mathrm{b}}$ \\
MDI & 100 & $0.58 \pm 0.17$ & $50.89 \pm 8.32$ & $49.11 \pm 8.32$ & 5.93 \\
\hline
\end{tabular}

Descriptive data are expressed as the mean \pm standard deviation. ${ }^{\mathrm{a}} \mathrm{P}<0.001$ and ${ }^{\mathrm{b}} \mathrm{P}<0.01$ versus MDI group. CSII, continuous subcutaneous insulin infusion; MDI, multiple daily injection.

Table III. Comparison of efficacy between the CSII and MDI groups for 12 weeks.

\begin{tabular}{lcc}
\hline & \multicolumn{2}{c}{ Group } \\
\cline { 2 - 3 } Parameter & MDI & CSII \\
\hline HbAlc & $10.79 \pm 1.42$ & $10.86 \pm 1.36$ \\
Baseline (\%) & $7.51 \pm 1.28$ & $7.11 \pm 1.32^{\mathrm{a}}$ \\
End-point $(\%)$ & $-3.47 \pm 1.53$ & $-3.75 \pm 1.48$ \\
Change $(\%)$ & $<0.005$ & $<0.005$ \\
P-value & & \\
FBG & $8.68 \pm 3.32$ & $8.61 \pm 3.12^{\mathrm{a}}$ \\
Baseline $(\mathrm{mmol} / \mathrm{l})$ & $6.85 \pm 1.26$ & $6.76 \pm 1.13$ \\
End-point $(\mathrm{mmol} / \mathrm{l})$ & $-1.83 \pm 2.61$ & $-1.85 \pm 2.36$ \\
Change $(\mathrm{mmol} / \mathrm{l})$ & $<0.005$ & $<0.005$ \\
P-value & & \\
2-h PBG & $15.60 \pm 5.71$ & $15.42 \pm 4.78^{\mathrm{a}}$ \\
Baseline $(\mathrm{mmol} / \mathrm{l})$ & $9.95 \pm 2.16$ & $9.87 \pm 2.63$ \\
End-point $(\mathrm{mmol} / \mathrm{l})$ & $-5.65 \pm 4.48$ & $-5.56 \pm 4.15$ \\
Change $(\mathrm{mmol} / \mathrm{l})$ & $<0.005$ & $<0.005$ \\
P-value & & \\
\hline
\end{tabular}

Descriptive data are expressed as the mean \pm standard deviation. ${ }^{a} \mathrm{P}>0.05$ versus MDI group. PBG, postprandial blood glucose; $\mathrm{FBG}$, fasting blood glucose; CSII, continuous subcutaneous insulin infusion; MDI, multiple daily injection.

Table IV. Comparison of insulin doses upon achievement of good blood glucose control after 12 weeks.

\begin{tabular}{lrr}
\hline & \multicolumn{2}{c}{ Group } \\
\cline { 2 - 3 } Parameter & \multicolumn{1}{c}{ MDI } & \multicolumn{1}{c}{ CSII } \\
\hline Rate (IU/kg/day) & $0.47 \pm 0.19$ & $0.39 \pm 0.23^{\mathrm{a}}$ \\
Basal insulin dose (\%) & $50.88 \pm 8.42$ & $69.23 \pm 6.89^{\mathrm{a}}$ \\
Bolus insulin dose (\%) & $49.12 \pm 8.11$ & $30.77 \pm 6.99^{\mathrm{a}}$ \\
\hline
\end{tabular}

Descriptive data are expressed as the mean \pm standard deviation. ${ }^{\mathrm{a}} \mathrm{P}<0.001$ versus MDI group. CSII, continuous subcutaneous insulin infusion; MDI, multiple daily injection.

however, good glycemic control was reached in both groups and no statistical differences were observed in FBG levels, $2 \mathrm{~h}$
Table V. Comparison of blood glucose levels at different times and blood glucose fluctuations after 12 weeks.

\begin{tabular}{lcc}
\hline & \multicolumn{2}{c}{ Group } \\
\cline { 2 - 3 } Parameter & MDI & CSII \\
\hline Rate (IU/kg/day) & $0.47 \pm 0.19$ & $0.39 \pm 0.23^{\mathrm{a}}$ \\
Basal insulin dose (\%) & $50.88 \pm 8.42$ & $69.23 \pm 6.89^{\mathrm{a}}$ \\
Bolus insulin dose (\%) & $49.12 \pm 8.11$ & $30.77 \pm 6.99^{\mathrm{a}}$ \\
Blood glucose levels (mmol/l) & & \\
Fasting & $6.17 \pm 0.71$ & $6.06 \pm 0.51$ \\
2 h after breakfast & $8.96 \pm 1.51$ & $8.76 \pm 1.11$ \\
2 h after lunch & $9.23 \pm 1.21$ & $9.05 \pm 0.91$ \\
2 h after supper & $8.59 \pm 1.19$ & $8.19 \pm 0.89$ \\
3:00 a.m. & $6.26 \pm 0.89$ & $6.21 \pm 0.61$ \\
Blood glucose fluctuation (mmol/l) & $0.23 \pm 0.06$ & $0.19 \pm 0.04^{\mathrm{b}}$ \\
\end{tabular}

Descriptive data are expressed as the mean \pm standard deviation. ${ }^{\mathrm{a}} \mathrm{P}<0.001$ and ${ }^{\mathrm{b}} \mathrm{P}<0.01$ versus MDI group.

post-breakfast, $2 \mathrm{~h}$ post-lunch or $2 \mathrm{~h}$ post-supper blood glucose levels or blood glucose levels at 3:00 a.m. between the two groups (Table V). Blood glucose fluctuations for the CSII group were lower than those for the MDI group $(\mathrm{P}<0.001)($ Table V). Insulin requirements decreased $19.12 \pm 2.3 \%$ after 12 weeks for good glycemic level control in the two groups.

\section{Discussion}

Blood glucose daily fluctuations contribute to oxidative stress, which can cause long-term complications in patients with diabetes (29). Avoiding glucose fluctuations in patients with diabetes is an emerging challenge (30). The data presented indicate that fluctuations in daily blood glucose were narrower for patients undergoing CSII therapy than for those undergoing MDI therapy. The results were consistent with the theory that basal insulin adjustment using CSII therapy in patients with diabetes provides less variable blood glucose levels than long-acting insulin (31). The variability in blood glucose control appears to be particularly significant with regard to long-acting insulin. However, no difference was detected in daily blood fluctuations between CSII and MDI therapies in a previous study of patients with T2DM (32).

Hypoglycemia is one of the main factors for patients with diabetes requiring insulin to achieve tight glycemic control 
and a reduced likelihood of complications. No statistically significant differences in nocturnal hypoglycemic episodes and hypoglycemia were detected between the two groups in the present study $(\mathrm{P}>0.05)$, suggesting that the safety of the MDI therapy may be comparable to that of CSII therapy in subjects with T2DM.

The data presented in this study showed that, in patients with T2DM treated with insulin therapy for 12 weeks, the total daily dose of insulin in the MDI group was significantly greater than that in the CSII group. The basal dose regulation in the MDI group was not convenient and the mutation variation rates of subcutaneous absorption of bolus insulin aspart and basal insulin glargine were considerably greater in the MDI group than those in the CSII group. Three factors contribute to PBG in diabetes: Increases in glycogen output, FBG and absorption of intestinal glucose. In general, increasing the range of $2 \mathrm{~h}-\mathrm{PBG}$ depends upon increasing the gastrointestinal glucose absorption and glycogen output. However, gastrointestinal glucose absorption is the same in patients with T2DM and healthy subjects (31) Basal insulin may restrict the glycogen output (33) by decreasing both pre-meal blood glucose and FBG levels and partially restraining postprandial hyperglycemia. Although decreasing PBG may be treated using basal insulin, a lower pre-meal insulin dose is still required as a supplement to control PBG. The pre-meal insulin dose may only restrain the absorption-related increase in glucose and some of the output of glycogen. A lower pre-meal dose may decrease the additive effect of the basal dose, therefore avoiding the requirement for adjustments to be made the basal dose. The study by Suzuki et al (33) indicated that an increase in basal insulin dose may be an effective method to control HbA1c and FBG in patients with T2DM and showed the dominance of basal insulin treatment.

Due to the adjustment therapy method used in the present study, the total daily bolus insulin dose of the CSII group was $30.87 \pm 6.99 \%$; this contrasted with the total daily bolus dose used in the treatment of Korean patients $(64.11 \pm 12.10 \%)(34)$. In the present study, the mean total daily dose of insulin was $31.68 \pm 8.88 \mathrm{IU}(0.48 \pm 0.11 \mathrm{IU} / \mathrm{kg} / \mathrm{day})$ for the 100 patients in the CSII group, while in a study of 46 Indian patients with T2DM the daily insulin dose was 44.0 23.7 IU/day (18). In the present study, the total daily bolus dose (17.55 \pm 3.52 IU) of the MDI group subjects comprised $49.11 \pm 8.32 \%$ of the total daily dose, and the mean total daily dose of insulin was $37.12 \pm 10.18 \mathrm{IU} /$ day $(0.58 \pm 0.17 \mathrm{IU} / \mathrm{kg} /$ day $)$; these values were both higher than those of the CSII group subjects. In all subjects, the mean total daily dose of insulin was $34.86 \pm 9.76$ IU and the dose of insulin per unit body weight was $0.53 \pm 0.17 \mathrm{IU} / \mathrm{kg} /$ day. The total daily bolus and basal insulin doses were $13.75 \pm 5.01$ and $21.12 \pm 7.91 \mathrm{IU}(40.07 \pm 11.88$ and $59.93 \pm 11.88 \%$ of total daily dose), respectively. These values differed from those in a previous report where the basal/total daily ratio of insulin was $0.23 \pm 0.08$ and the mean daily dose of insulin was 38.22 \pm 14.92 IU/day (35). From studying 200 patients with T2DM and other related research reports $(21,22,34)$, pre-meal and basal dose proportions have been shown to be associated with the total insulin dose. Pre-meal blood glucose levels can be controlled by preferentially regulating basal insulin, making the required total daily dose of insulin lower.
After 12 weeks of application, MDI-treated patients with T2DM had a higher total insulin dose requirement and hypoglycemia incidence and took longer to achieve the targeted glycemic control compared with the CSII-treated patients. Following CSII treatment in patients with T2DM, decreases in bolus dose and increases in basal insulin dose can form an effective method to achieve good glycemic control with a lower total daily dose. However, where factors exist to prevent the use of therapy with insulin pumps, once daily glargine at bedtime combined with aspart administration at each of the three meals should be an effective alternative.

\section{Acknowledgements}

This study was supported by the Shandong Province Science and Technology Development Plan (2012YD18019). The authors would like to thank all the staff working at the Departments of Endocrinology and Clinical Medicine in the Linyi People's Hospital.

\section{References}

1. Lindholm A, McEwen J and Riis AP: Improved postprandial glycemic control with insulin aspart. A randomized double-blind cross-over trial in type 1 diabetes. Diabetes Care 22: 801-805, 1999

2. Anderson JH Jr, Brunelle RL, Koivisto VA, et al: Reduction of postprandial hyperglycemia and frequency in hypoglycemia in IDDM patients on insulin-analog treatment. Multicenter Insulin Lispro Study Group. Diabetes 46: 265-270, 1997.

3. Raskin P, Klaff L, Bergenstal R, et al: A 16-week comparison of the novel insulin analog insulin glargine (HOE901) and NPH human insulin used with insulin lispro in patients with type 1 diabetes. Diabetes Care 23: 1666-1671, 2000.

4. Lepore M, Pampanelli S, Fanelli C, et al: Pharmacokinetics and pharmacodynamics of subcutaneous injection of long-acting human insulin analog glargine, NPH insulin, and ultralente human insulin and continuous subcutaneous infusion of insulin lispro. Diabetes 49: 2142-2148, 2000.

5. Pickup J and Keen H: Continuous subcutaneous insulin infusion at 25 years: evidence base for the expanding use of insulin pump therapy in type 1 diabetes. Diabetes Care 25: 593-598, 2002.

6. Reynolds LR: Reemergence of insulin pump therapy in the 1990s. South Med J. 93: 1157-1161, 2000.

7. Bode BW, Steed RD and Davidson PC: Reduction in severe hypoglycemia with long-term continuous subcutaneous insulin infusion in type 1 diabetes. Diabetes Care 19: 324-327, 1996.

8. Bode BW, Weinstein R, Bell D, et al: Comparison of insulin aspart with buffered regular insulin and insulin lispro in continuous subcutaneous insulin infusion: a randomized study in type I diabetes. Diabetes Care 25: 439-444, 2002.

9. Bode B and Strange P: Efficacy, safety, and pump compatibility of insulin aspart used in continuous subcutaneous insulin infusion therapy in patients with type 1 diabetes. Diabetes Care 24: 69-72, 2001.

10. Tsui E, Barnie A, Ross S, et al: Intensive insulin therapy with insulin lispro: a randomized trial of continuous subcutaneous insulin infusion versus multiple daily insulin injection. Diabetes Care 24: 1722-1727, 2001.

11. Elbein SC, Wegner K and Kahn SE: Reduced beta-cell compensation to the insulin resistance associated with obesity in members of caucasian familial type 2 diabetic kindreds. Diabetes Care 23: 221-227, 2000.

12. Yang W, Lu J, Weng J, et al; China national Diabetes and Metabolic Disorders Study Group: Prevalence of diabetes among men and women in China. N Engl J Med 362: 1090-1101, 2010.

13. An YL, Gao Y, Zhu Q, et al: Features of insulin secretion and insulin resistance in newly diagnosed type 2 diabetes of different body mass indices Zhonghua Yi Xue Za Zhi 24: 256-260, 2008 (In Chinese).

14. Juan CC, Fang VS, Kwok CF, et al: Exogenous hyperinsulinemia causes insulin resistance, hyperendothelinemia, and subsequent hypertension in rats. Metabolism 48: 465-471, 1999. 
15. Bruttomesso D, Pianta A, Crazzolara D, et al: Continuous subcutaneous insulin infusion (CSII) in the Veneto region: efficacy, acceptability and quality of life. Diabet Med 19: 628-634, 2002.

16. Weissberg-Benchell J, Antisdel-Lomaglio J and Seshadri R: Insulin pump therapy: a meta-analysis. Diabetes Care 26: 1079-1087, 2003.

17. Bruttomesso D, Crazzolara D, Maran A, et al: In Type 1 diabetic patients with good glycaemic control, blood glucose variability is lower during continuous subcutaneous insulin infusion than during multiple daily injections with insulin glargine. Diabet Med 25: 326-332, 2008.

18. Jothydev Kesavadev J, Balakrishnan S, Ahammed S, Jothydev S: Reduction of glycosylated hemoglobin following 6 months of continuous subcutaneous insulin infusion in an Indian population with type 2 diabetes. Diabetes Technol Ther 11: 517-21, 2009.

19. Torres I, Ortego J, Valencia I, et al: Benefits of continuous subcutaneous insulin infusion in type 1 diabetes previously treated with multiple daily injections with once-daily glargine and pre-meal analogues. Exp Clin Endocrinol Diabetes 117: 378-385, 2009.

20. Phillip M, Battelino T, Rodriguez H, et al; European Society for Paediatric Endocrinology; Lawson Wilkins Pediatric Endocrine Society; International Society for Pediatric and Adolescent Diabetes; American Diabetes Association; European Association for the Study of Diabetes: Use of insulin pump therapy in the pediatric age-group: consensus statement from the European Society for Paediatric Endocrinology, the Lawson Wilkins Pediatric Endocrine Society, and the International Society for Pediatric and Adolescent Diabetes, endorsed by the American Diabetes Association and the European Association for the Study of Diabetes. Diabetes Care 30: 1653-1662, 2007.

21. Monami M, Lamanna C, Marchionni N and Mannucci E: Continuous subcutaneous insulin infusion versus multiple daily insulin injections in type 2 diabetes: a meta-analysis. Exp Clin Endocrinol Diabetes 117: 220-222, 2009.

22. Kesavadev J, Balakrishnan S, Ahammed S and Jothydev S: Reduction of glycosylated hemoglobin following 6 months of continuous subcutaneous insulin infusion in an Indian population with type 2 diabetes. Diabetes Technol Ther 11: 517-521, 2009.

23. Carotenuto M, Esposito M, Parisi L, et al: Depressive symptoms and childhood sleep apnea syndrome. Neuropsychiatr Dis Treat 8: 369-373, 2012.

24. Verrotti A, Agostinelli S, D'Egidio C, et al: Impact of a weight loss program on migraine in obese adolescents. Eur J Neurol 20 394-397, 2013
25. Carotenuto M, Santoro N, Grandone A, et al: The insulin gene variable number of tandem repeats (INS VNTR) genotype and sleep disordered breathing in childhood obesity. J Endocrinol Invest 32: 752-755, 2009.

26. Carotenuto M, Bruni O, Santoro N, et al: Waist circumference predicts the occurrence of sleep-disordered breathing in obese children and adolescents: a questionnaire-based study. Sleep Med 7: 357-361, 2006.

27. Hoogma RP, Hammond PJ, Gomis R, et al; 5-Nations Study Group: Comparison of the effects of continuous subcutaneous insulin infusion (CSII) and NPH-based multiple daily insulin injections (MDI) on glycaemic control and quality of life: results of the 5-nations trial. Diabet Med 23: 141-147, 2006.

28. Workgroup on Hypoglycemia, American Diabetes Association: Defining and reporting hypoglycemia in diabetes: a report from the American Diabetes Association Workgroup on Hypoglycemia. Diabetes Care 28: 1245-1249, 2005.

29. Marcovecchio ML, Lucantoni M and Chiarelli F: Role of chronic and acute hyperglycemia in the development of diabetes complications. Diabetes Technol Ther 13: 389-394, 2011.

30. Ceriello A and Ihnat MA: 'Glycaemic variability': a new therapeutic challenge in diabetes and the critical care setting. Diabet Med 27: 862-867, 2010.

31. Bragd J, von Döbeln A, Lins PE, et al: Basal insulin substitution with glargine or continuous subcutaneous insulin infusion in adult type 1 diabetes patients-a randomized controlled trial. Diabetes Technol Ther 12: 689-693, 2010.

32. Tone A, Iseda I, Higuchi C, et al: Comparison of insulin detemir and insulin glargine on glycemic variability in patients with type 1 and type 2 diabetes. Exp Clin Endocrinol Diabetes 118: 320-324, 2010.

33. Suzuki D, Toyoda M, Kondo M, et al: Efficacy of long-acting insulin analog insulin glargine at high dosage for basal-bolus insulin therapy in patients with type 2 diabetes. Tokai J Exp Clin Med 37: 35-40, 2012.

34. Cai X, Han X, Luo Y and Ji L: Analysis of insulin doses of Chinese type 2 diabetic patients with intensive insulin treatment. PLoS One 7: e38962, 2012.

35. Kelley D, Mokan M and Veneman T: Impaired postprandial glucose utilization in non-insulin-dependent diabetes mellitus. Metabolism 43: 1549-1557, 1994. 